\title{
Essential role of using virtual pyeloscopy in the diagnosis of small satellite renal pelvic tumour in solitary kidney patient
}

\author{
Pal Bata, MD; ${ }^{*}$ David Laszlo Tarnoki, MD; ${ }^{*}$ Adam Domonkos Tarnoki, MD; Zsolt Domjan, MD, PhD; ${ }^{\dagger}$ \\ Istvan Buzogany, MD; ${ }^{+}$Viktor Berczi, MD, PhD, DSc ${ }^{*}$
}

*Department of Radiology and Oncotherapy, Semmelweis University, Budapest; †Department of Urology, Péterfy Hospital, Budapest, Hungary

Cite as: Can Urol Assoc J 2012;6(5):E195-E198. htrp://dx.doi.org/10.5489/cuaj.11160

\section{Abstract}

Virtual pyeloscopy (VP) plays an important role in the organ-sparing nephroscopic tumour resection and traditional pole resection, especially when a fibreoptic ureteroscopic examination cannot be performed. A 67-year-old man developed macroscopic hematuria. Abdominal computed tomography (CT) and cystoscopy revealed a lower calix renal stone and a 20-mm lower pyelon mass suggesting transitional cell cancer. An additional small satellite lesion in the pelvico-ureteral junction was supposedly present. Three-phase MultiDetector CT with VP showed a solitary 3 to $4-\mathrm{mm}$ renal pelvic lesion beyond the known calix stone and lower pole mass. In our case, VP played an important role in the organ-sparing nephroscopic tumour resection and traditional pole resection.

\section{Introduction}

Painless and symptom-free hematuria is a very important sign of urologic malignancy. Diagnostic possibilities for the evaluation of the upper renal tract tumours (pelvis and ureter) include contrast-enhanced (CE) computed tomography (CT), semirigid (traditional) or flexible ureterorenoscopy (URS) or virtual pyeloscopy (VP). Advantages of multidetector CT (MDCT) are excellent spatial and contrast resolution, delineation of the exact borders of the lesions, detection of the surrounding tissue and organs state (local or distant metastases), and reproducibility and post-processing possibilities, like virtual imaging. There are a few reports on the role of VP in the diagnosis of upper urinary tract tumours. ${ }^{1-5}$ $\mathrm{VP}$ can play an important role in organ-sparing nephroscopic tumour resection and traditional partial nephrectomy, especially in cases where a fibreoptic ureteroscopic examination cannot be performed (e.g., patients with ureteral strictures or high-risk, solitary kidney patients).

\section{Case report}

A 67-year-old male presented on August 2007 with a 6-month history of recurrent hematuria. He had a history of abdominal surgery for trauma at age 14, and right-sided nephrectomy for calculous pyelonephritis in 1994. Three CT scans and an intravenous pyelography were done, after which a renal stone occupying the lower calyx was identified. In addition, the examinations confirmed a 20-mm parenchymal mass of the lower pyelon (Fig. 1, Fig. 2). The morphology and contrast-enhancement of the lesion, as well as the patient's history, strongly suggested transitional cell cancer (TCC). The same CT examination revealed a small 3-mm satellite lesion at the pelvico-ureteral junction (Fig. 3, Fig. 4). The recommended magnetic resonance imaging (MRI) was later not performed because the patient developed a large coagulated hematuria and bladder tamponade, resulting in his admission to the urology department on May 2008. Bladder catheter was inserted, vitamin K1 (konakion), rutozide trihidrate (rutascorbin), etamsylate (dicynone) and vitamin C were given, after which no further hematuria was observed.

To characterize the lesions, we performed an additional diagnostic test. Semirigid or flexible URS was not considered a first step because of the limited irrigation possibilities, the risk of tumour spread, the presence of solitary kidney and the age of this high-risk patient. Three-phase (30s, 70s, 300s) MDCT (Philips Brilliance 16, Best, The Netherlands) was performed (120 mL Optiray [Mallinckrodt Inc., Hazelwood, MO] $350 \mathrm{mgl} / \mathrm{mL}, 3 \mathrm{~mL} / \mathrm{sec}$ flow) with a collimation of $16 \times 1.5 \mathrm{~mm}, 50 \%$ overlap, $2 \mathrm{~mm}$ slice thickness. Volume rendered technique and software (ENDO 3D, Philips, Best, The Netherlands) was used to produce VP images. VP confirmed a solitary satellite 3 to 4 -mm large renal pelvic lesion beyond the known calix stone and lower pole mass (Fig. 5, Fig. 6). Biopsy of this lesion was not possible in this case partially because of its size, the presence of the solitary kidney and the suggested diagnosis by VP. Urine cytology indicated inflammatory cells without suspicion of malignancy. 


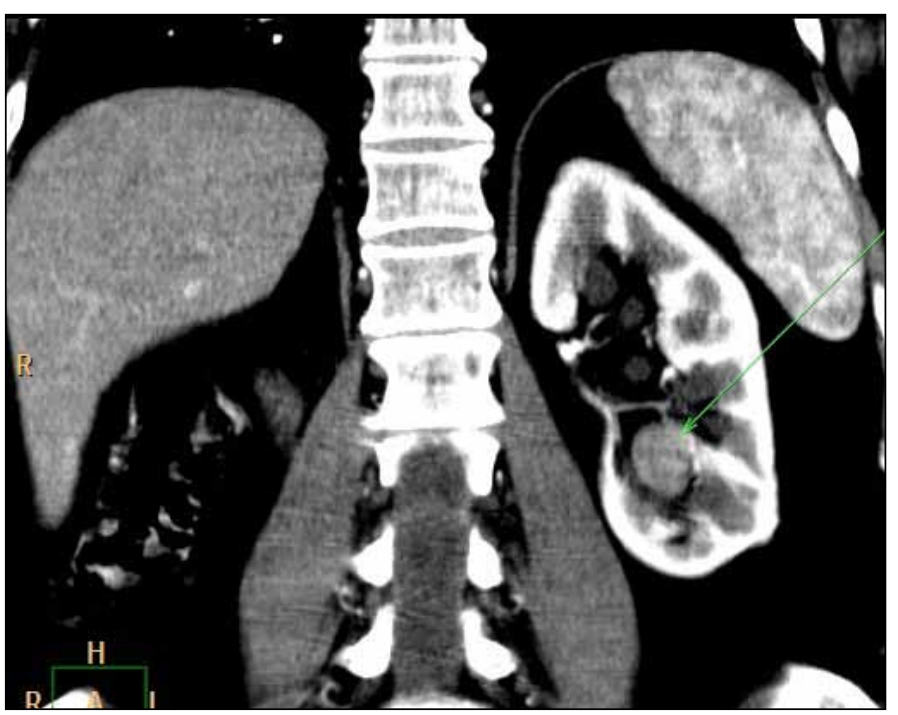

Fig. 1. Transitional cell carcinoma (lower pole mass) in the left lower renal pelvis (arrow). Contrast enhanced computed tomography image, corticomedullar phase, coronal view.

Considering the presence of a solitary kidney and the bifocal lesions, it was decided to perform the organ-sparing percutaneus nephroscopic stone elimination by ultrasonic lithotripsy and uretero-pelvic tumour resection, while the lower calyx tumor was managed via open partial lower pole nephrectomy. Using a resectoscope (Olympus OES Pro, A22021A, 27 Fr.) via percutaneous nephrostomy tract, the papillary tumour could be completely removed without leaving macroscopic residuals or pelvic perforation. Histopathology of the small pyelo-ureteral lesion demonstrated a pT1 grade I TCC without invasion. The $20-\mathrm{mm}$ lower pole mass was deemed a pT1 grade II TCC with lymphatic invasion. A single local instillation mitomycin $\mathrm{C}$ and Bacillus Calmette-Guérin was administered after insertion of a double J stent, but it was not continued because of the presence of fever.

Control examination by cystoscopy, ultrasonography, urine sediment and urine cytology following the operation every 3 months did not reveal signs of recurrence (a full-length video of VP before the operation can be watched: http://www.radi. sote.hu/tudomanyos.html under "Virtual pyeloscopy").

\section{Discussion}

Endoscopic procedures represent the gold standard for the investigation of urothelium. Diagnostic indications include filling defects on urography, unilateral hematuria, positive urinary cytology and surveillance after tumour resection. ${ }^{1}$ These procedures are uncomfortable, so sedation or anesthesia may be required; complications include creation of a false passage, fever, sepsis, ureteral perforation and delayed stricture formation. ${ }^{6}$ Enlarged prostate and ureteral strictures may cause difficulties during the procedures.

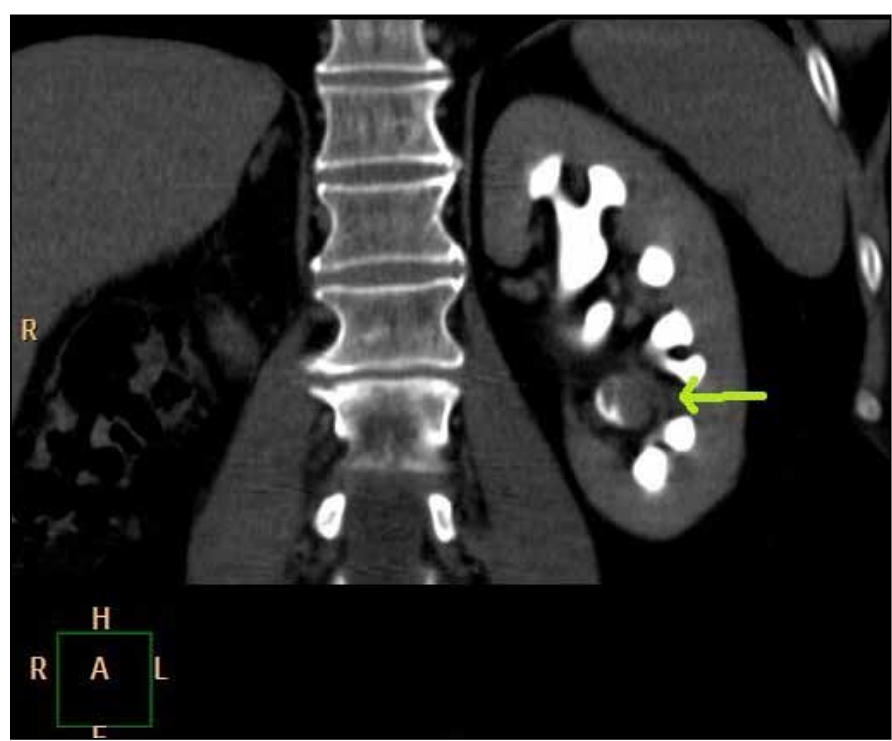

Fig. 2. Transitional cell carcinoma (lower pole mass) in the left renal pelvis. Contrast enhanced computed tomography image, nephrographic phase, coronal view.

In this particular case, VP was an invaluable tool in finding the concomitant malignancy of the solitary kidney. Our technology has made it possible to virtually simulate endoscopies based on helical CT or MR datasets. VP is a volumerendering technique that simulates endoscopic views. A high visual gradient is required between the visceral lumen and wall structures to allow differentiation of these structures, depending on the selection of a suitable threshold value. The endoscopic effect is produced by perspective rendering along a path within the dataset. During the post-processing method, the viewer can interactively change the location, viewing direction and the visual field of the VP. Orientation can be established from multiplanar reconstructions and from the virtual endoscopic image itself.

The advantages of VP compared to URS are its non-invasiveness that eliminates the risk of perforation or any other mechanical injury, preclusion of the need of anesthesia, and its ability to provide precise and high-resolution information on parenchymal lesions, retroperitoneal and perivesical lymph node and tumour recurrence in the urinary bladder or contralateral kidney. The only disadvantage is its lack of opportunity for biopsy, which is true of any virtual technique.

There are very few reports on VP of upper urinary tract tumors. In 1999, a Japanese publication presented the results of 32 patients, on whom VP was used. Surface rendering techniques facilitated non-invasive visualization of the complex morphology of renal pelvic carcinomas. VP was superior to CT in detecting infiltration and pedunculation, which was shown to be advantageous when endourological procedures were planned. ${ }^{2}$ The study reported that the CE-CT scan demonstrated $83 \%$ sensitivity compared to surgi- 


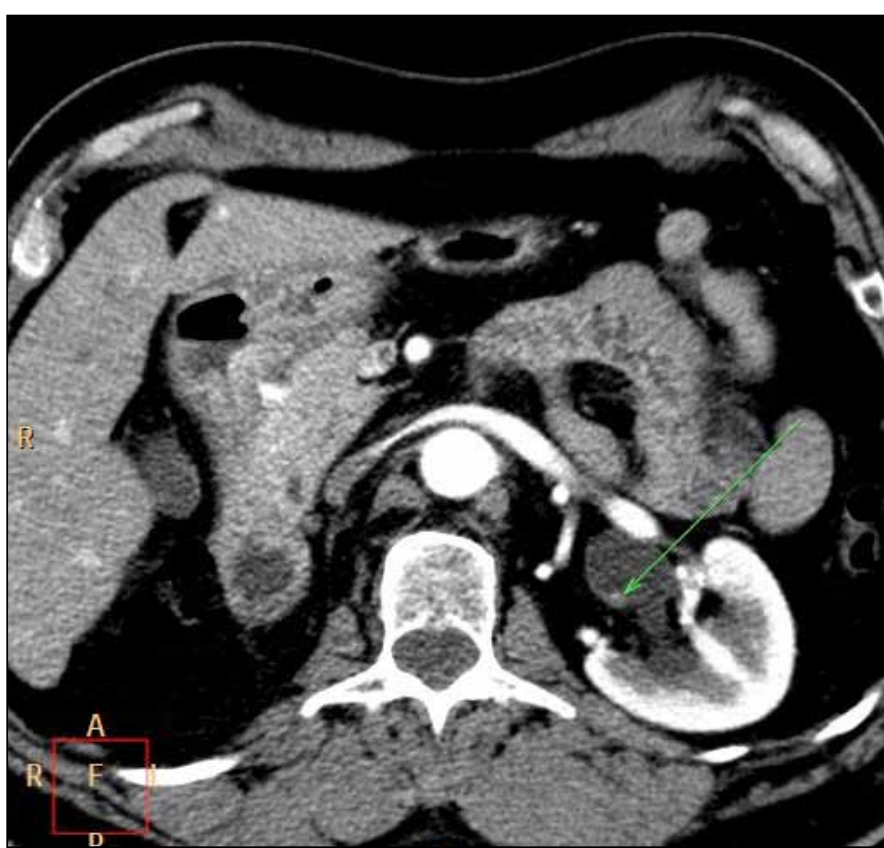

Fig. 3. Computed tomography image of the small lesion in the pyelo-ureteral junction (arrow). Contrast enhanced computed tomography image, corticomedullar phase, axial view.

cal histopathology findings in 23 patients. CT scan integrated with VP imaging technique had a $92 \%$ sensitivity in the detection of renal cell carcinoma. CT ureteroscopy done on a subgroup of 16 patients demonstrated its usefulness in visualizing the complex morphology of ureteral tumour and its ability to distinguish ureteral strictures from tumours. ${ }^{3}$ On this small cohort of patients, $81 \%$ sensitivity and $100 \%$ specificity were reported for detecting ureteral tumour and carcinoma. ${ }^{3}$ In another small cohort, virtual pyelography showed findings strongly indicative of malignancy, which was missed by CT-urography in two of these patients. ${ }^{1}$

Three-dimensional CT pyelography was useful in demonstrating calculus position and spatial relationships of the collecting system before percutaneous nephrostolithotomy. ${ }^{5}$ Not only polypoid cancer, but also stones, could be evaluated and localized properly by VP. This technique helps the planning of the endoscopic procedures as presented in our case.

VP should be integrated in CT-urography for a complete evaluation of the upper urinary tract in patients with suspected tumour, thus limiting the need for fibreoptic ureteroscopic examination. ${ }^{1}$ In our practice, it is currently used only as a problem-solving tool for difficult cases. To establish a successful diagnosis using $\mathrm{VP}$, collaboration with the referring physician (e.g., urologists) is crucial. Application of the correct methods and techniques for pyeloureteral imaging, proper consideration of the artifacts, and knowledge of the limitations of the techniques are important for the radiologist.

Virtual endoscopy trainings for example on a virtual reality (VR) simulator have been shown to improve the performance of VR endoscopic tasks by novice endoscopists. ${ }^{7}$

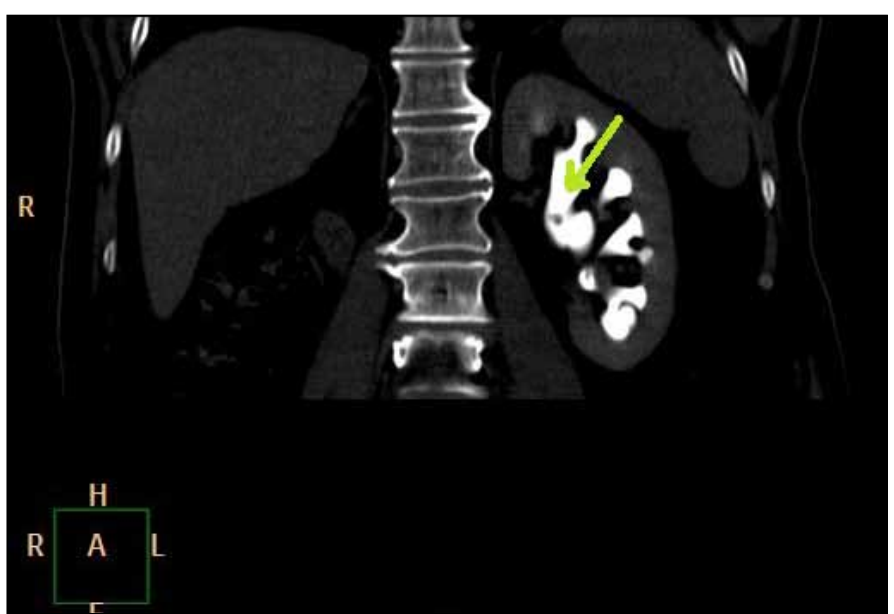

Fig. 4. Computed tomography image of the small lesion in the pyelo-ureteral junction (arrow). Contrast enhanced computed tomography image, nephrographic phase, coronal view.

However, VR training is unable to override the impact of clinical training, although it may help shorten the learning curve during the earlier period of learning the techniques.

The advantages of VP compared to URS include its noninvasiveness, no need of anesthesia, no risk of perforation or any other mechanical injury, and precise, high resolution information concerning parenchymal lesions. In addition, as VP is integrated to a CT scan examination, additional information concerning the surrounding areas (e.g., retroperitoneal and perivesical lymph node status, recurrence of TCC in the urinary bladder or contralateral kidney, if present) can be also obtained. Finally, the CT scan with integrated VP is more cost-effective than an additional endoscopic procedure. VP saves costs compared to the optical or conventional counterpart, since usually no sedation, patient preparation or even hospitalization is required. VP needs no extra investigation, the only additional cost is about an extra 15 to 20 minutes for the radiologist for the postprocessing evaluation.

The disadvantages are the lack of possibility for biopsy, and the lack of information about colour and texture of the lesion. In case of renal impairment or history of allergic reactions to contrast media, CE-CT is not recommended and therefore VP will not be possible in these cases. The allergic reactions to contrast media are uncommon, ranging from $5 \%$ to $12 \%$ of intravascular injections with ionic agents to $1 \%$ to $3 \%$ with nonionic lower-osmolarity agents. ${ }^{8}$ Mild adverse effects, including nausea, vomiting, urticaria, feeling of warmth with injection, higher pulse rate, are common. Moderate allergic reactions (e.g., bronchospasm, mild laryngeal edema, vasovagal reactions) are less common and commonly need treatment. Severe potential life-threatening side effects (e.g., severe bronchospasm, severe laryngeal edema, loss of consciousness, seizures, cardiac arrest or complete cardiovascular collapse) occur 1 in 130000 cases..$^{9}$ Our patient did not experience side effects during the CE-CT study. 


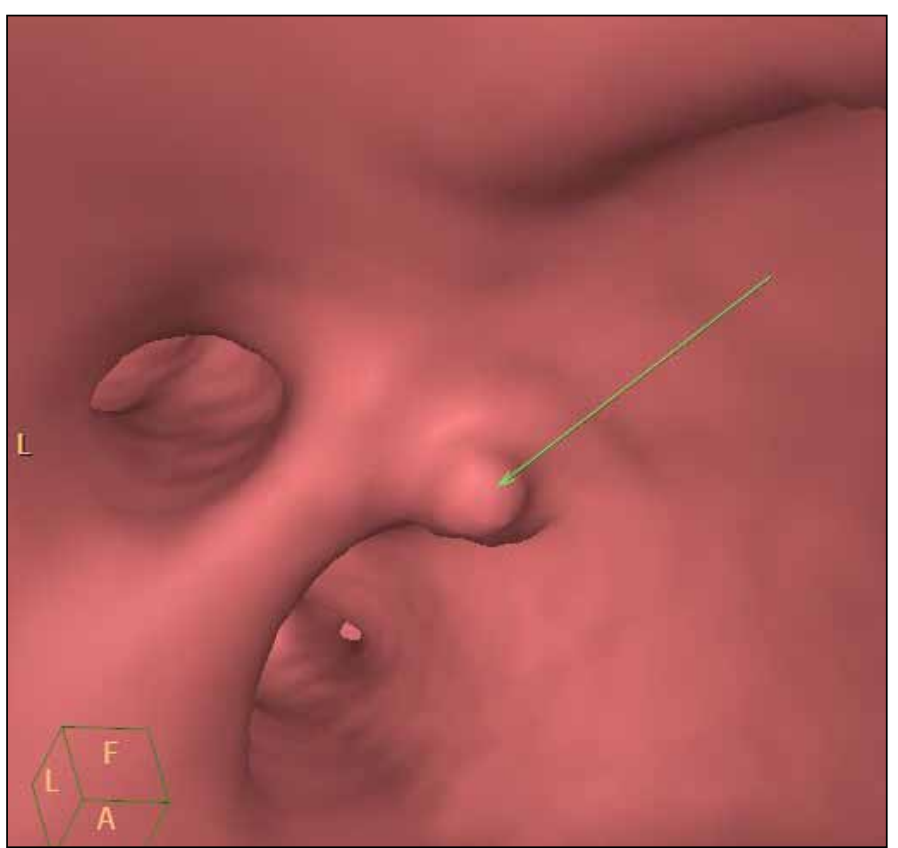

Fig. 5. Virtual pyeloscopy 3D computed tomography image of the small lesion in the pyelo-ureteral junction (arrow) (view of the pelvis)

Further studies with a greater number of patients are recommended to confirm the sensitivity and specificity of this new technology. An ideal research design should compare satellite tumors found on VP and the autopsy findings.

\section{Conclusion}

VP played an important role in the organ-sparing nephroscopic tumour resection and traditional pole resection. VP performed during the multiphase MDCT examination without the need of further radiation is a noninvasive method that is suggested to be integrated in CT-urography for a complete evaluation of the upper urinary tract in patients with suspected tumors. The need for fibreoptic ureteroscopic examination is limited by VP. During VP, there is no need for sedation or anesthesia; complications include creation of false passage, fever, sepsis, ureteral perforation and delayed stricture formation. In cases of extremely enlarged prostate or ureteral stricture if the ureteral access is difficult for endoscopic procedures, VP can be performed. VP is an alternative in high-risk cases patients with solitary kidney.

Competing interests: None declared.

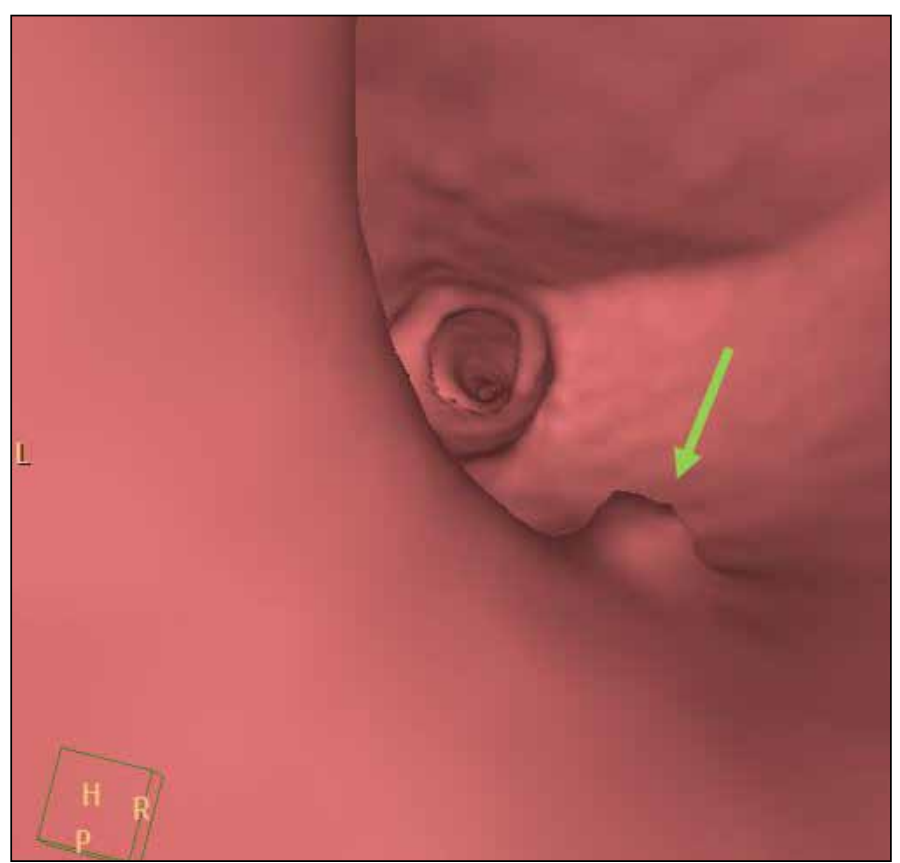

Fig. 6. Virtual pyeloscopy 3D computed tomography image of the small lesion in the pyelo-ureteral junction (arrow); view from the pelvis towards the ureter

\section{References}

1. Battista G, Sassi C, Sciavina R, et al. Computerized tomography virtual endoscopy in evalutation of upper urinary tract tumors: initial experience. Abdom Imag 2009;34:107-12. http://dx.doi.org/10.1007/ s00261-008-9387-5

2. Takebayashi S, Hosaka M, Takase K, et al. Computerized tomography nephroscopic images of renal pelvic carcinoma. J Urol 1999;162:315-8. http://dx.doi.org/10.1016/S0022-5347(05)68548-5

3. Takebayashi S, Hosaka M, Kubota Y, et al. Computerized tomographic ureteroscopy for diagnosing ureteral tumors. J Urol 2000;163:42-6. http://dx.doi.org/10.1016/S0022-5347(05)67968-2

4. Fielding JR, Borland D, Lee KH, et al. Virtual pyeloscopy using volumetric depth peeling. Acad Radiol 2006;13:759-63. http://dx.doi.org/10.1016/..acra.2006.02.042

5. Patel U, Walkden RM, Ghani KR, et al. Three-dimensional CT pyelography for planning of percutaneous nephrostolithotomy: accuracy of stone measurement, stone depiction and pelvicalyceal reconstruction. Eur Radiol 2009;19:1280-8. http://dx.doi.org/10.1007/s00330-008-1261-x

6. Harmon WJ, Sershon PD, Blute ML, et al. Ureteroscopy: current practice and long-term complications. J Urol 1997;157:28-32. http://dx.doi.org/10.1016/S0022-5347(01)65272-8

7. Boyle E, $0^{\prime}$ Keeffe $D A$, Naughton $P A$, et al. The importance of expert feedback during endovascular simulator training. J Vasc Surg 2011;54:240-8. http://dx.doi.org/10.1016/i.jvs.2011.01.058

8. American College of Radiology Committee on Drugs and Contrast Media. Manual on Contrast Media. 5th Edition. Reston, VA: American College of Radiology; 2004.

9. Bettmann MA, Heeren T, Greenfield A, et al. Adverse events with radiographic contrast agents: results of the SCVIR Contrast Agent Registry. Radiology 1997;203:611-20.

Correspondence: Prof. Viktor Berczi, Department of Radiology and Oncotherapy, Semmelweis University, 78/A Ulloi street, Budapest, 1082, Hungany; fax: +36-1-2780368; berczi@hotmail.com

This paper has been peer-reviewed. 